\title{
A Robust Algorithm Against Actuator Saturation using Integral Sliding Mode and Composite Nonlinear Feedback
}

\author{
B.Bandyopadhyay ${ }^{*}$ Fulwani Deepak ${ }^{* *}$ Y.J.Park ${ }^{* * *}$ \\ * IDP in Systems and Control Engineering, Indian Institute of \\ Technology,Bombay,India (Tel: 091-22-25767889; e-mail: \\ bijnan@sc.iitb.ac.in). \\ ** IDP in Systems and Control Engineering,Indian Institute of \\ Technology,Bombay,India (e-mail: fulwani@iitb.ac.in) \\ *** Department of Mechanical Engineering, KAIST, Taejon 305-701, \\ South Korea (e-mail: yjpark@kaist.ac.kr)
}

\begin{abstract}
Integral sliding mode(ISM) based composite nonlinear feedback(CNF) tracking controller to track step signal is proposed. The proposed controller combines advantages of CNF controller like quick response without overshoot and robustness of ISM controller. Integral sliding mode is used along with CNF controller to reject disturbances and track nominal trajectory. Actuator saturation effect is considered and the stability of overall system is guaranteed with saturated actuator. Chattering is reduced by use of sigmoid function and non-linear switching gain. Effectiveness of the proposed scheme is demonstrated by simulation results.
\end{abstract}

Keywords: Robust control;Anti-windup;Tracking

\section{INTRODUCTION}

Performance specifications of any system are often specified in terms of step response. For a quick response damping ratio should not be too large because large damping ratio increases settling time. However too small damping ratio gives rise to overshoot and ringing. Thus, most of design schemes make a trade-off between these two transient performance indices. Z. Lin et al. [1998] proposed the seminal idea of composite non linear feedback(CNF). In CNF initially damping ratio is kept very low to ensure quick response and as output approaches the set point system is made highly damped to avoid overshoot by nonlinear feedback. M. C. Turner et al. [2000] first proposed general form of this concept for high order SISO and MIMO systems with state feedback in which authors have generalized the concept with some restrictions which eventually leads to exclusion of systems which were originally considered by Z. Lin et al. [1998]. This concept is further extended by Chen et al. [2003] for more general class of systems with only output feedback and it is also proved that CNF controller is better than time optimal controller for asymptotic tracking case. From practical point of view asymptotic tracking is more important. K. Peng et al. [2005] proposed enhanced CNF controller with integral action in forward path. Enhanced CNF controller is not robust for all types of disturbances and integral action may lead to so called integral windup phenomena. In G. Cheng et al. [2007] proposed robust CNF controller based on disturbance estimation. Here constant disturbance is assumed which is observed by an observer and the effect of constant bias is compensated. But in actual system time varying disturbances are inevitable. To the best of our knowledge no attempts have been made to make CNF controller robust against time varying disturbance. To reject the disturbance, structure of CNF controller should not be changed otherwise the actual motive of CNF controller will be lost. Proposed ISM-CNF based controller retains actual structure of CNF controller while rejecting disturbances and thus it ensures invariance.

Drazenovic [1969] established that the controlled system in the sliding mode is completely robust against certain class of parameter variations and external disturbances satisfying matching condition. Basic theory on sliding mode control can be found in textbook written by Edward et al. [1999] and survey paper by Gao et al. [1993]. In conventional sliding mode control system motion is constrained to lie in ( $n-m)$ dimensional manifold with suitable control action. Here $\mathrm{m}$ is the number of inputs and $\mathrm{n}$ is the order of the system. With discontinuous control action system trajectory is forced to lie on sliding manifold. Motion of trajectory from initial condition towards sliding surface until it hits sliding surface is called reaching phase. During the reaching phase controlled system is not robust and even matched disturbances can affect the system. To solve this problem Utkin et al. [1996] proposed integral sliding mode concept. An integral term is added in sliding manifold, this guarantees that the system trajectories will start in the manifold right from the beginning, therefore reaching phase is eliminated. The main idea is to define control law as a sum of nominal control and a discontinuous control. Nominal control takes care of nominal plant dynamics and discontinuous control rejects the disturbances. Nominal control can be of any form which is able to follow reference trajectory within a given accuracy. In this work we have taken CNF controller as the nomi- 
nal controller along with ISM controller as discontinuous controller to reject the disturbance. Hence it is possible to avail of the advantages offered by CNF controller and rejecting disturbances through integral sliding mode control.

\subsection{Motivation}

The CNF controller works well when there is no disturbances in the plant. But disturbances are inevitable in actual plant environment. So far no attempts have been made to ensure invariance against time varying disturbance. However in actual plant time varying disturbance may be present. It is illustrated through example that time varying disturbance deteriorates performance significantly. Even stability can not be guaranteed in face of disturbance and saturation when plant is open loop unstable. To achieve invariance against disturbances another robust control strategy should be combined with CNF technique without sacrificing performance of controlled system. Integral sliding mode naturally allows to combine other techniques with sliding mode control. Sliding mode provides invariance against class of disturbance which satisfy matching condition. With integral sliding mode we can also avoid amplification of unmatched perturbation. This motivates us to propose a new integral sliding mode based CNF controller for a general class of step signal tracking problem.

\subsection{Structure of Paper}

In the next section we present CNF controller for the plant with matched uncertainty. We have modified the linear part of CNF controller to avoid saturation with ISM controller. Section 3 presents integral sliding mode with CNF controller and we prove stability of system with this combined control law. Example and simulation results are presented in Section 4. We will conclude our work in Section 5 .

\section{COMPOSITE NONLINEAR FEEDBACK CONTROL}

In this section we review CNF controller and modify linear controller part of CNF so that ISMC can be combined without actuator saturation. Discussion in this Section will go parallel with Chen et al. [2003] with the modification that the maximum amplitude of reference signal to be tracked is affected by the maximum value of uncertainty. Consider the nominal plant with uncertainty,

$$
\begin{aligned}
& \dot{x}=A x+B \operatorname{sat}(u)+B \rho_{d}(x, t) . \\
& y=C x .
\end{aligned}
$$

Where $x \in \Re^{n}, u \in \Re, y \in \Re$ are , respectively, the state, input,and controlled output of the system.A,B, $C$ matrices of appropriate dimensions. $\rho_{d}(x, t)$ is state bounded matched uncertainty and only bounds are known. Function sat: $\mathrm{R} \rightarrow \mathrm{R}$ represents actuator saturation defined as,

$$
\operatorname{sat}(u)=\operatorname{sign}(u) \times \operatorname{Min}\left\{u_{\max },\|u\|\right\} \text {. }
$$

Where $u_{\max }$ is maximum value of input. Here we have considered single input case to show effectiveness of the concept in a simple manner. However it should be noted that the concept can be extended for multi input case without any modifications. We make following assumptions

(1) $\operatorname{Pair}(\mathrm{A}, \mathrm{B})$ is controllable.

(2) $(\mathrm{A}, \mathrm{B}, \mathrm{C})$ has no zeros at $s=0$.

Control Objective:Controlled output y should track input command $\mathrm{r}$, in the presence of disturbances and uncertainty in the plant and without experiencing large overshoot.

For system (1) linear feedback control law is given as,

$$
u_{L}=F x+G_{1} r .
$$

$\mathrm{F}$ is chosen such that $(\mathrm{A}+\mathrm{BF})$ is an asymptotically stable matrix, and it should have small damping ratio. Matrix $G_{1}$ is defined as

$$
G_{1}=-\left[C(A+B F)^{-1} B\right]^{-1} .
$$

This can be found by finding d.c gain from y to $r$. When $\mathrm{y}$ tracks $\mathrm{r}$, $\mathrm{x}$ will take new steady state value $x_{e}$ which is given as

$$
x_{e}=-(A+B F)^{-1} B G_{1} r=G_{e} r
$$

where $G_{e}=-(A+B F)^{-1} B G_{1}$ This can be found by finding d.c gain between $\mathrm{x}$ and $\mathrm{r}$. Let us make co-ordinate transformation as

$$
\tilde{x}=x-x_{e}
$$

where $\tilde{x}$ is the error between actual state and desired state. The linear control law becomes

$$
u_{L}=F \tilde{x}+H r
$$

where $H=\left[I-F(A+B F)^{-1} B\right] G_{1}$.

The maximum value of command input $\mathrm{r}$ that can be followed is determined as follows.

Lemma 1. Let the real symmetric matrix $P>0$ be the solution of the following Lyapunov equation

$$
(A+B F)^{T} P+P(A+B F)=-W,
$$

for positive definite matrix W. Such a $\mathrm{P}$ exist, because $(\mathrm{A}+\mathrm{BF})$ is a stable matrix.

let $c_{\delta}>0$ be the largest positive scalar satisfying the following condition

$$
|F x| \leq u_{\max }(1-\delta) \forall x \in X_{\delta}=\left\{x \mid x^{T} P x \leq c_{\delta}\right\},
$$

where $\delta \in(0,1)$. Then the linear feedback control law (4) makes system output to track asymptotically a step command input of amplitude $r$, when initial error and $r$ satisfy

$$
\begin{gathered}
\forall \tilde{x} \in X_{\delta}, \\
|H r| \leq \delta_{1} u_{\text {max }},
\end{gathered}
$$

where, $0 \leq \delta_{1}<\delta$ and $\left|\left(\delta-\delta_{1}\right) u_{\max }\right|=\rho_{d}(x, t)_{\max }$ maximum bound of disturbance.

Remark:1 In the statement of this lemma we have redistributed control efforts $u_{\max } \delta$ on disturbance and $\mathrm{Hr}$ unlike Chen et al. [2003]. The redistribution is done to ensure stability of closed loop system with uncertainty. This will reduce the maximum amplitude of reference 
signal to be tracked depending on bounds of uncertainty. Proof: by construction

$$
|F \tilde{x}+H r| \leq \epsilon u_{\max }
$$

where $\epsilon=1-\delta+\delta_{1}$ and $0<\epsilon<1$.

By design, linear control law does not saturate input. Therefore from (1) and (7) we can write the error dynamics as follows

$$
\tilde{\dot{x}}=(A+B F) \tilde{x}+A x_{e}+B H r .
$$

It is straight forward to see that $A x_{e}+B H r=0$ so the error dynamics becomes

$$
\tilde{\dot{x}}=(A+B F) \tilde{x},
$$

which shows error dynamics is stable. Furthermore it shows $X_{\delta}=\left\{x \mid x^{T} P x \leq c_{\delta}\right\}$ is an invariant set of closed loop system (15).

Remark 2: In above lemma to prove stability, linear controller is constrained to remain $u_{L} \leq \epsilon u_{\max }$. This will put upper limit on the amplitude of reference signal to be tracked. Following equations should satisfy to track step signal of amplitude $\mathrm{r}$

$$
\begin{gathered}
|r| \leq \sqrt{c_{\delta}\left(G_{e}^{T} P G_{e}\right)^{-1}}, \\
|H r| \leq \delta_{1} .
\end{gathered}
$$

The inequality(16) can be proved as follows

$$
\begin{aligned}
x^{T} P x & \leq c_{\delta}, \\
\Rightarrow x_{e}^{T} P x_{e} & \leq c_{\delta}, \\
\Rightarrow\left(G_{e} r\right)^{T} P\left(G_{e} r\right) & \leq c_{\delta}, \\
\Rightarrow r^{T} G_{e}^{T} P G_{e} r & \leq c_{\delta}, \\
\Rightarrow r^{T} r G_{e}^{T} P G_{e} & \leq c_{\delta},
\end{aligned}
$$

because $\mathrm{r}$ is a scalar. Manipulating the above equation we get

$$
|r| \leq \sqrt{c_{\delta}\left(G_{e}^{T} P G_{e}\right)^{-1}}
$$

\subsection{Nonlinear Control}

$(\mathrm{A}+\mathrm{BF})$ is having low damping factor. To increase the damping factor non-linear control is added with linear controller and it is given as follows

$$
u_{N}=\Psi(r, x) B^{T} P\left(x-x_{e}\right),
$$

where $\Psi(r, x)$ is any non positive function locally lipschitz in y. Non linear control is used to change the system's closed loop damping ratio as the output approaches step command input. Initial choice for selection of $\Psi(r, x)$ given by Z. Lin et al. [1998] is modified to get extra degree of freedom by Chen et al. [2003]. Non linear function should be chosen such that it changes from 0 to $-\beta$ where $\beta>0$. One possible choice of function as suggested by Chen et al. [2003] as

$$
\Psi(r, x)=-\frac{\beta}{1-e^{-1}}\left(e^{-\left|1-\left(y-y_{0}\right) /\left(r-y_{0}\right)\right|}-e^{-1}\right),
$$

where $y_{0}=y(0)$, and $\beta$ is used as a tuning parameter.
From equation (4) and (18) we can write combined linear and non-linear control law as

$$
u_{0}=u_{L}+u_{N}=F x+G_{1} r+\Psi(r, x) B^{T} P(x-x e) .
$$

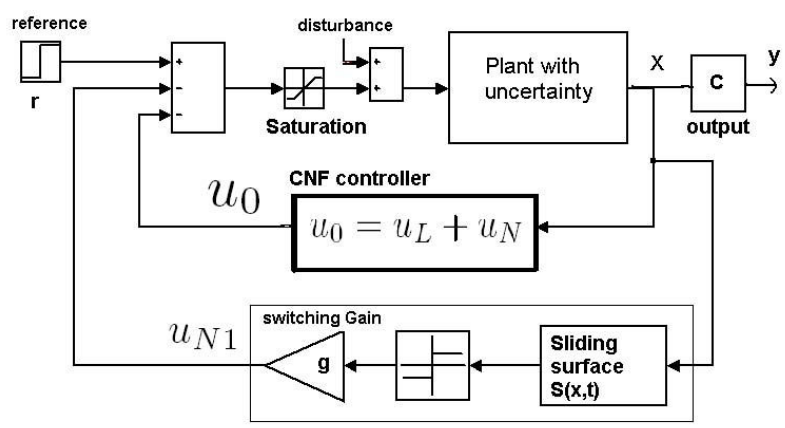

Fig. 1. Block diagram with combined ISM-CNF control law

\section{INTEGRAL SLIDING MODE CONTROL}

Controller given by eqn.(20) does not provide robust closed loop performance. We propose integral sliding mode based controller with CNF controller to make overall control system robust. In this section we design another nonlinear component which is added with controller given by equation (20) to provide robust closed loop performance. Integral sliding manifold proposed by Fridman et al. [2006] as

$$
s(x, t)=G\left\{x(t)-x_{0}-\int_{0}^{t}\left(A x+B u_{0}\right) d \tau\right\} .
$$

In above sliding surface equation $u_{0}$ can be of any form. The $u_{0}$ when substituted in nominal plant(i.e eqn.(1) without disturbance term $\left.\rho_{d}(x, t)\right)$ gives nominal trajectory. In the above eqn. $\int_{0}^{t}\left(A x+B u_{0}\right) d \tau$ represents desired trajectory and $x(t)$ is actual trajectory. So sliding surface can be seen as the difference between actual and the desired trajectory projected on G. The difference occurs due to disturbances. By using appropriate control action this difference can be nullified and actual trajectory tracks the required one.

Our objective is to find discontinuous control law to force sliding motion on $\mathrm{s}(\mathrm{x}, \mathrm{t})=0$. Let this discontinuous control is $u_{N 1}$. Therefore actual control law becomes

$$
u=u_{0}+u_{N 1}=u_{L}+u_{N}+u_{N 1} .
$$

Here discontinuous control $u_{N 1}$ required for enforcing sliding motion is added with CNF controller. Figure 1 shows block diagram with ISM-CNF control law. To find discontinuous control law we construct Lyapunov function as

$$
V(x, t)=0.5 s^{T}(x, t) s(x, t) .
$$

By using equations (1),(21) and (22) we can write 


$$
\begin{aligned}
\dot{V}(x, t) & =\left[G \dot{x}-G\left(A x+B u_{0}\right)\right]^{T} s(x, t) \\
& =\left[G B u_{N 1}+G B \rho_{d}(x, t)\right]^{T} s(x, t) \\
& =\left[u_{N 1}+\rho_{d}(x, t)\right]^{T}(G B)^{T} s(x, t) .
\end{aligned}
$$

Usual choice for discontinuous control is

$$
u_{N 1}=-M(x, t) \operatorname{sign}\left((G B)^{T} s(x, t)\right) .
$$

Where $\mathrm{M}(\mathrm{x}, \mathrm{t})$ is positive definite function and calculated based on maximum disturbance bounds. With this discontinuous control law we can get

$$
\dot{V}(x, t)<0 .
$$

Remark3: In the above proof we have not considered actuator saturation. But by construction $u_{L}$ is less than $u_{\max }$ and discontinuous control is replaced by equivalent control $\left(u_{e q}\right)$ as proposed by Utkin et al. [1996] for analysis purpose. We can find $u_{e q}=-\rho_{d}(x, t)$. So maximum value of $u_{e q}$ is $\rho_{\text {dmax }}(x, t)$. By construction, $u_{L}+\rho_{\text {dmax }}(x, t) \leq u_{\max }$. So when the nonlinear component of CNF controller $u_{N}$ added with $u_{0}$ it causes actuator saturation. Next we find closed loop error dynamics for system(1) with input (22). From eqns. (1),(22) and (7) we can write the error dynamics as

$$
\begin{aligned}
\dot{\tilde{x}} & =(A+B F) \tilde{x}+B w+B \rho_{d}(x, t) \\
& =(A+B F) \tilde{x}+B g,
\end{aligned}
$$

where,

$$
\begin{aligned}
g & =w+\rho_{d}, \\
w & =\operatorname{sat}\left(F \tilde{x}+H r+u_{N}+u_{N 1}\right)-F \tilde{x}-H r .
\end{aligned}
$$

We will replace $u_{N 1}$ with its equivalent value for the analysis purpose, and equivalent value of $u_{N 1}$ is given as

$$
\left(u_{N 1}\right)_{e q}=u_{e q}=-\rho_{d}(x, t) .
$$

As we stated in remark 2 addition of $u_{N}$ with $u_{L}$ and $u_{N 1}$ creates saturation at input. By design contribution from other components is less than $u_{\max }$. In next theorem we will prove stability of closed loop system (1) with control input $(22)$.

Theorem 1 For the system in (1) output obtained by the application of the control proposed in (22) will track the desired constant reference trajectory asymptotically. In the proof of theorem we consider that the amplitude of reference signal is restricted by (16) and (17).

Proof: For error dynamics equation (14), Define Lyapunov function as

$$
\begin{aligned}
V & =\tilde{x}^{T} P \tilde{x} . \\
\Rightarrow \dot{V} & =-\tilde{x}^{T} W \tilde{x}+2 \tilde{x}^{T} P B g .
\end{aligned}
$$

Our aim is to ensure $\dot{V}$ should be negative definite. Here $\mathrm{g}$ is the only variable parameter. Now we calculate $\dot{V}$ for three different cases.

Case:1 If

therefore,

$$
\left|F x+H r+u_{N 1}+u_{N}\right| \leq u_{\max }
$$

$$
\begin{aligned}
g & =w+\rho_{d}(x, t), \\
& =F x+H r+u_{N 1}+u_{N}-F x-H r+\rho_{d}(x, t), \\
& =u_{N}, \\
& =\Psi(r, x) B^{T} P \tilde{x} .
\end{aligned}
$$

Because $u_{N 1}+\rho_{d}(x, t)=0$. It implies,

$$
\dot{V}=-\tilde{x}^{T} W \tilde{x}+2 \Psi(r, h) \tilde{x}^{T} P B B^{T} P \tilde{x} \leq-\tilde{x}^{T} W \tilde{x}
$$

Case:2 if

$$
\left|F x+H r+u_{N 1}+u_{N}\right|>u_{\max }
$$

By construction, $\left|F x+H r+\rho_{d}(x, t)\right| \leq u_{\max }$ so

$$
g=u_{\max }-F x-H r-\rho_{d}(x, t) .
$$

Therefore

$g>0$ and $u_{N}>u_{\max }-F x-H r+u_{N 1}>0$. Which implies that $\tilde{x}^{T} P B<0$. Hence

$$
\dot{V}=-\tilde{x}^{T} W \tilde{x}+2 \tilde{x}^{T} P B w \leq-\tilde{x}^{T} W \tilde{x} .
$$

Case:3 If

$$
\left|F x+H r+u_{N 1}+u_{N}\right| \leq-u_{\max }
$$

therefore,

$$
\begin{aligned}
g=-u_{\max }-F x-H r+\rho_{d}(x, t) \\
=-u_{\max }-F x-H r-u_{N 1} \\
\Rightarrow g<0 \\
\Rightarrow u_{N}<u_{\max }-F x-H r-u_{N 1}<0 \\
\Rightarrow \tilde{x}^{T} P B>0 \\
\Rightarrow 2 \tilde{x}^{T} P B g<0
\end{aligned}
$$

therefore

$\dot{V} \leq-\tilde{x}^{T} W \tilde{x}$.

Therefore in all case when $\mathrm{x}$ and $\mathrm{r}$ satisfy conditions (11) and (12) we have shown that $\dot{V} \leq-\tilde{x}^{T} W \tilde{x}$ when $\tilde{x} \in X_{\delta}$ This implies

$$
\lim _{t \rightarrow \infty} x(t)=x_{e}
$$

Therefore

$$
\lim _{t \rightarrow \infty} h(t)=C x_{e}=r .
$$

Above theorem proves the stability of closed loop system with combined input from CNF law and ISM law.

\section{EXAMPLE AND SIMULATION RESULTS}

In this section we study normalized pendulum (double integrator) with disturbance. Consider linear uncertain plant of double integrator

$$
\left[\begin{array}{c}
\dot{x_{1}} \\
\dot{x_{2}}
\end{array}\right]=\left[\begin{array}{ll}
0 & 1 \\
0 & 0
\end{array}\right]\left[\begin{array}{l}
x_{1} \\
x_{2}
\end{array}\right]+\left[\begin{array}{l}
0 \\
1
\end{array}\right] \operatorname{sat}(u)+d
$$




$$
y=\left[\begin{array}{ll}
1 & 0
\end{array}\right]\left[\begin{array}{l}
x_{1} \\
x_{2}
\end{array}\right]
$$

Where $\mathrm{d}$ is the disturbance and in this simulation we will take it as

$$
d=\left[\begin{array}{c}
0 \\
k x_{1} x_{2} \sin \left(x_{1}\right) u_{1}(t-\tau)
\end{array}\right]
$$

where $u_{1}(t-\tau)$ is unit step signal delayed by $\tau$ period. For this system our control objective is that state $x_{1}$ should track unit step as fast as possible without having overshoot. Maximum value of input is taken as $u_{\max }=1$. CNF controller proposed in Chen et al. [2003] for this plant is given as,

$$
u_{L}=[-6.5-1] x+6.5 r
$$

$$
u_{N}=-\left(e^{\|1-y\|}-0.36788\right)\left[\begin{array}{ll}
1.4481 & 10.8609
\end{array}\right]\left(x-\left[\begin{array}{l}
1 \\
0
\end{array}\right]\right)
$$

Total control effort becomes

$$
u_{0}=u_{L}+u_{N}
$$

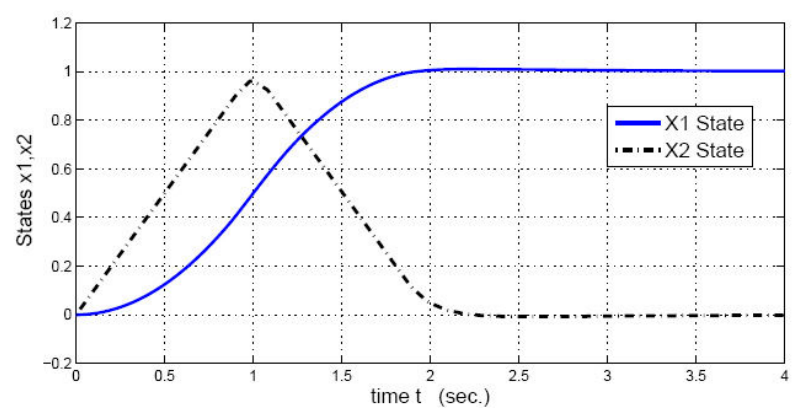

Fig. 2. Plot with CNF controller without disturbance

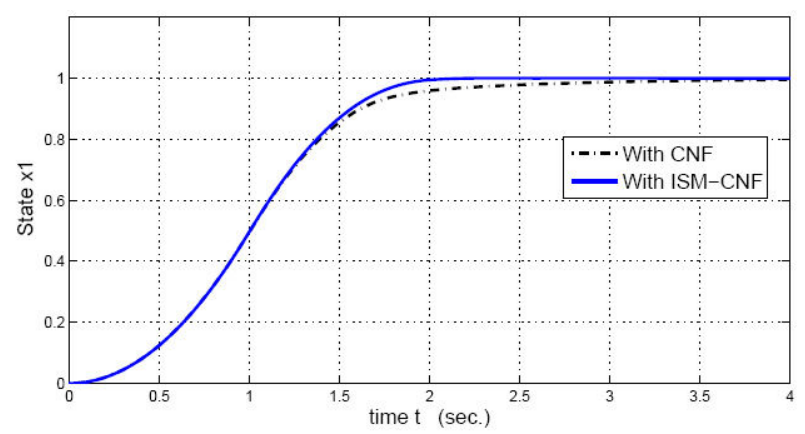

Fig. 3. Solid line shows performance with ISM-CNF controller and dotted line shows performance with CNF controller alone

Now we will design integral sliding mode control law. Above law is given just for comparison with new ISMCNF based control law. We are comparing our results with original CNF law because there is no robust algorithm available to reject time varying perturbations. ISM law can be designed as

$$
u_{N 1}(x, t)=-\eta|s|^{\alpha}\left|x_{2}\right| \operatorname{sign}\left((G B)^{T} s(x, t)\right) .
$$

Above law is similar to power rate reaching law specified in Gao et al. [1993]. Use of sign function results in very

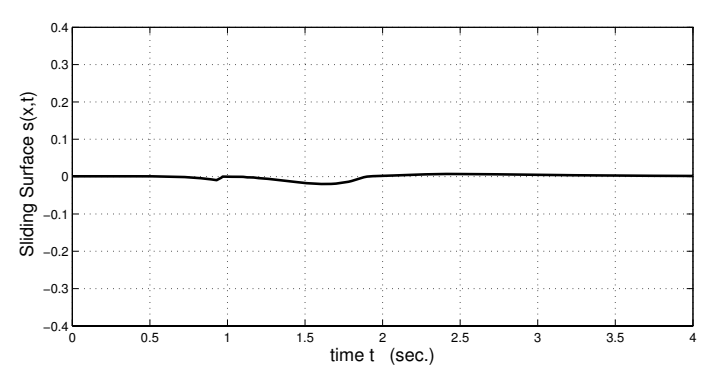

Fig. 4. Plot of sliding surface $\mathrm{s}(\mathrm{x}, \mathrm{t})$

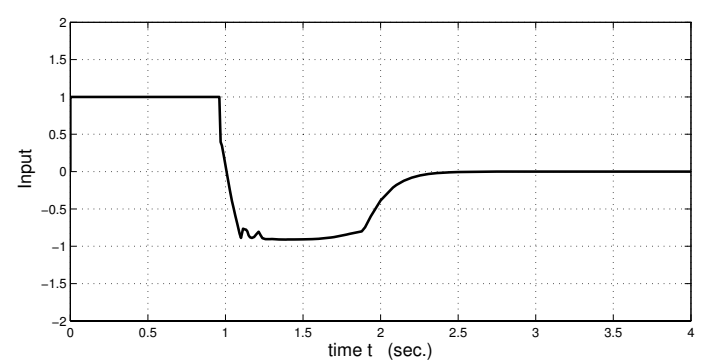

Fig. 5. Plot of input $u$

Table 1. Controller parameters for simulation

\begin{tabular}{|r|r|}
\hline Parameter & Value \\
\hline$\eta$ & 23.77 \\
$\alpha$ & 0.5 \\
$\theta$ & 0.002 \\
$\tau$ & 0.2 sec. \\
$k$ & 0.4 \\
\hline
\end{tabular}

high chattering so sigmoid function is used in place of sign function in actual simulation to reduce chattering so control law becomes

$$
u_{N 1}(x, t)=-\eta|s|^{\alpha}\left|x_{2}\right| \frac{\left((G B)^{T} s(x, t)\right)}{\left\|\left((G B)^{T} s(x, t)\right)\right\|+\theta} .
$$

Where $\theta$ is a small positive number which decides width of band where sliding surface is constrained. So switching is done when it crosses the band.

As recently proved in Fridman et al. [2006] to avoid amplification of unmatched perturbation $G$ should be generalized inverse of input matrix B. So $\mathrm{G}$ becomes,

$$
G=B^{+}=\left[\begin{array}{ll}
0 & 1
\end{array}\right] \text {. }
$$

Sliding surface can be computed by eqn (21). Simulation is carried out for the plant with following parameters.

Figure-2 shows CNF controller without disturbance and $x_{1}$ tracks unit step in 2 seconds, here disturbance $\mathrm{d}=0$. When we add disturbance term in plant with CNF controller performance deteriorates. It tracks reference within 3.5 seconds which is clear from Figure-3. With ISM-CNF controller Figure-3 shows that $x_{1}$ tracks reference within 2 seconds as if there is no disturbance in the plant. Therefore ISM-CNF controller rejects disturbance completely and controlled plant behaves as a nominal plant with nominal controller. Figure- 4 shows sliding surface which is a measure of deviation of actual trajectory from ideal nominal trajectory without disturbance. It is seen from Figure-4 
that sliding surface remains close to zero. Figure- 5 shows there is no chattering in the input because of use of sigmoid function in place of sign function.

\section{CONCLUSION}

ISM-CNF controller provides quick response without any overshoot. ISM-CNF controller does not have any switching element unlike time optimal control. Change of gain is smooth. ISM-CNF strategy gives better performance than time optimal control for asymptotic tracking. With ISMCNF controller we get invariance. Controlled plant rejects any disturbance which satisfy matching condition. ISMCNF controller is able to avoid amplification of unmatched perturbation by the universal choice of G matrix. Stability of system is assured in spite of saturation at actuator. Effectiveness of this new ISM-CNF scheme to achieve invariance against disturbances is proved.

\section{REFERENCES}

John. Y. Hung, W. Gao. Variable Structure Control: A Survey. IEEE Transaction on Ind. Electronics, vol. 40, no. 1,pages 2-22, 1993.

Drazenovic The invariance conditions in variable structure systems. Automatica,vol. 5, no. 3,pages 287-295, 1969.

Zongli Lin, Meir Pachter, Siva Banda. Towards improvement of tracking performance-nonlinear feedback for linear systems. International Journal of Control, vol. 70, no. 1,pages 1-11, 1998.

M. C. Turner, I Postlethwaite, D. J. Walker. Nonlinear tracking control for multivariable constrained input linear systems. International Journal of Control, vol. 73, no. 12,pages 1160-1172, 2000.

Ben. M. Chen, T. H. Lee, K. Peng,V.Venkataramanan. Composite nonlinear feedback control for linear systems with input saturation: theory and an application. IEEE Trans. on Automatic Control, vol. 48, no. 3,pages 427439, 2003.

Ben. M. Chen, G. Cheng, T. H. Lee. Modeling and compensation of nonlinearities and friction in a micro hard disk servo system with nonlinear control. IEEE Trans. on Control Systems Technology, vol. 13, no. 5,pages 708-721, 2005.

Guoyang Cheng, Kemao Peng. Robust composite nonlinear feedback control with application to a servo positioning system. IEEE Transaction on Ind. Electronics, vol. 54, no. 2,pages 1132-1140, 2007.

Fernando Castanos, Leonid Fridman. Analysis and design of integral sliding manifolds for systems with unmatched perturbations. IEEE Trans. on Automatic Control, vol. 51, no. 5,pages 853-858, 2006.

V.I.Utkin, J. Shi. Integral sliding mode in systems operating under uncertainty conditions. Proceedings of the 35th conference on decision and control,pages 4591$4596,1996$.

C. Edward, S.K. Spurgeon. Sliding mode control: theory and application. Taylor and Francis, 1998. 